Port Acadie

Revue interdisciplinaire en études acadiennes

An Interdisciplinary Review in Acadian Studies

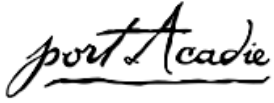

\title{
Mariaagélas, Pélagie-la-Charrette et le folklore acadien
}

\section{Carmen d'Entremont}

Numéro 22-23, automne 2012, printemps 2013

URI : https://id.erudit.org/iderudit/1014980ar

DOI : https://doi.org/10.7202/1014980ar

Aller au sommaire du numéro

\section{Éditeur(s)}

Université Sainte-Anne

ISSN

1498-7651 (imprimé)

1916-7334 (numérique)

Découvrir la revue

Citer cet article

d’Entremont, C. (2012). Mariaagélas, Pélagie-la-Charrette et le folklore acadien. Port Acadie, (22-23), 163-182. https://doi.org/10.7202/1014980ar

\section{Résumé de l'article}

Maillet a souvent insisté sur le caractère essentiellement oral de ses textes, ainsi que sur sa personnalité de conteuse, s'opposant par là au rôle normal de l'écrivain. Plusieurs ont donc affirmé que son oeuvre rend hommage à la tradition orale, ce qui s'est surtout fait en discutant de sa forme narrative. Or on a rarement envisagé le point de vue de l'ethnologie dans les nombreuses références à la culture populaire que Maillet emprunte directement au folklore acadien, et qui, pour l'étude de cette discipline, représente une richesse énorme. Il y a quarante ans, Maillet, par ses études doctorales, dégageait le caractère populaire de l'oeuvre rabelaisienne, afin de mesurer la richesse du folklore acadien. On peut maintenant considérer l'apport populaire de l'oeuvre mailletienne, ce que je propose à partir de deux romans, Mariaagélas et Pélagie-la-Charrette, à l'étude des légendes et des croyances superstitieuses.
Ce document est protégé par la loi sur le droit d'auteur. L'utilisation des services d'Érudit (y compris la reproduction) est assujettie à sa politique d'utilisation que vous pouvez consulter en ligne.

https://apropos.erudit.org/fr/usagers/politique-dutilisation/ 


\title{
Mariaagélas, Pélagie-la-Charrette et le folklore acadien
}

\author{
Carmen d'Entremont \\ Université Sainte-Anne
}

\begin{abstract}
Résumé
Maillet a souvent insisté sur le caractère essentiellement oral de ses textes, ainsi que sur sa personnalité de conteuse, s'opposant par là au rôle normal de l'écrivain. Plusieurs ont donc affirmé que son œuvre rend hommage à la tradition orale, ce qui s'est surtout fait en discutant de sa forme narrative. Or on a rarement envisagé le point de vue de l'ethnologie dans les nombreuses références à la culture populaire que Maillet emprunte directement au folklore acadien, et qui, pour l'étude de cette discipline, représente une richesse énorme. Il y a quarante ans, Maillet, par ses études doctorales, dégageait le caractère populaire de l'œuvre rabelaisienne, afin de mesurer la richesse du folklore acadien. On peut maintenant considérer l'apport populaire de l'œuvre mailletienne, ce que je propose à partir de deux romans, Mariaagélas et Pélagie-la-Charrette, à l'étude des légendes et des croyances superstitieuses.
\end{abstract}

La thèse doctorale d'Antonine Maillet, Rabelais et les traditions populaires en Acadie, illustre les origines médiévales de la tradition orale encore vivante chez le peuple acadien : «[...] l'Acadie a su conserver vivants, pendant plus de trois siècles cinq cents mots, une centaine de proverbes, une centaine d'images, une cinquantaine de jeux, plus combien de chansons, de croyances, de légendes et de contes » ${ }^{1}$. Arrivé au nouveau monde, ce folklore, transmis d'une génération à l'autre, s'est enrichi, s'est adapté et transformé selon les besoins et les valeurs. Au début des années 1970, au moment des études en ethnologie de Maillet à l'Université Laval, plusieurs chercheurs prétendaient que la tradition orale était en danger. Germain Lemieux, qui avait formulé cette crainte, voyait avec pessimisme l'avenir des enquêtes folkloriques; il allait même jusqu'à prédire, en 1972, que les enquêtes folkloriques seraient bientôt devenues impossibles : "Le folklore tire à sa fin; dans dix ans, le folkloriste ne sera pas ce qu'il est aujourd'hui, parce que les détenteurs de la tradition orale se seront tous tus pour toujours : la période d'enquêtes aura pris fin! $»^{2} \|$ y a donc eu une période de récupération : il fallait se hâter d'interroger les derniers témoins de ce passé! Maillet aussi avait senti cette urgence de la cueillette, surtout en Acadie : "[...] surtout, nous croyons à l'urgence

1. Antonine Maillet, Rabelais et les traditions populaires, Québec, Presses de l'Université Laval (PuL), 1980 [1 $1^{\text {re }}$ éd., 1971], p. 187.

2. Jean-Pierre Pichette, "Du bon vieux temps au patrimoine vivant », dans Jean-Pierre Pichette (dir.), L'Fuvre de Germain Lemieux, s.j. Bilan de l'ethnologie en Ontario français, Sudbury, Prise de parole et Centre franco-ontarien de folklore, 1993, p. 488. 
de traiter cette question parce que si Rabelais, lui, ne risque pas de disparaître, il n'en va pas ainsi de la littérature orale acadienne qui, par son caractère oral, sûrement, et peut-être son caractère acadien, repose sur un sable mouvant ${ }^{3}$. Ainsi, Maillet, qui se voyait dernière d'une race ${ }^{4}$, parcourait "les coins les plus retirés des provinces maritimes » dans le but de recueillir, "le plus vite possible, les épaves de ce dernier naufrage de la culture orale acadienne $»^{5}$. Voulant faire part de ses découvertes, elle présente le fruit de ses enquêtes folkloriques par l'entremise de ses écrits.

Comme le fait remarquer le littéraire René LeBlanc, Maillet a souvent insisté sur le caractère essentiellement oral de ses textes, ainsi que sur sa personnalité de conteuse, s'opposant par là au rôle normal de l'écrivain ${ }^{6}$. Plusieurs chercheurs ont donc affirmé que l'œuvre de Maillet rend hommage à la tradition orale, ce qui s'est surtout fait en discutant de sa forme narrative, particulièrement des trois niveaux diégétiques du roman Pélagie-la-Charrette ${ }^{7}$ qui, par leurs discours polyphoniques, controversés et simultanés, mettent en évidence le caractère mobile du récit oral. Or on a rarement pris en compte les multiples références à la culture populaire que Maillet emprunte directement au folklore acadien et qui, pour l'étude de l'ethnologie, représente une richesse énorme. Sheila Henderson montre qu'il y a dans le texte Pélagie-la-Charrette des allusions aux variantes de la littérature orale; selon elle, "le folklore est incorporé dans l'histoire comme une sorte de détente pour le lecteur ${ }^{8}$. Pour sa part, Thomas Brown suggère que Maillet a incorporé des éléments de la culture populaire à son roman dans le but de faire ressortir ses thèmes ${ }^{9}$. 11 y a

3. Antonine Maillet, Rabelais et les traditions populaires, op. cit., p. Ix.

4. Antonine Maillet, «Expériences d'écriture », dans Études françaises, vol. 12, n 1-2, 1976, p. 80.

5. $\quad$ Antonine Maillet, Rabelais et les traditions populaires, op. cit., p. 16.

6. René LeBlanc, "L'Oralité du style dans les romans d'Antonine Maillet », dans Revue d'histoire littéraire du Québec et du Canada français, Éditions de l'Université d’Ottawa, vol. 12, 1986, p. 36.

7. Voir, entre autres, Renatro Venâncio Henriques de Sousa, « Histoire et histoires dans Pélagie-la-Charrette : le récit oral comme sauvegarde de la mémoire collective ", dans Marie-Linda Lord (dir.), Lire Antonine Maillet à travers le temps et l'espace, Moncton, Institut d'études acadiennes, 2010, p. 125-142; Megessa O’Reilly, « Une écriture qui célèbre la tradition orale : Pélagie-la-Charrette d'Antonine Maillet ", dans Studies in Canadian Literature, vol.18, 1993, p. 118-127; René LeBlanc, op. cit., p. 35-49.

8. Sheila Henderson, "Le personnage de Bélonie et le folklore acadien ", dans Derrière la charrette de Pélagie : lecture analytique du roman d'Antonine Maillet, Pélagie-la-Charrette, contribution de Shirley Boudreau [et al.]; animation et rédaction, René LeBlanc, Pointe de l'Église (N.-É.), Presse de l'Université Sainte-Anne, 1984 , p. 92.

9. Thomas H. Brown, « Maintaining cultural traditions through oral history: Antonine Maillet's Pélagie-la-Charrette », dans MIFLC Proceedings, 1987, p. 71. 
quarante ans, Maillet, par ses études doctorales, dégageait le caractère populaire de l'œuvre rabelaisienne afin de mesurer la richesse du folklore acadien ${ }^{10}$. On peut maintenant considérer l'apport de ce folklore à l'œuvre mailletienne, ce que je propose, à partir de deux romans, Mariaagélas ${ }^{11}$ et Pélagie-la-Charrette ${ }^{12}$, par l'étude des légendes et des croyances superstitieuses.

\section{Légendes et croyances}

D’après les études en tradition orale, le $x_{1} x^{e}$ siècle fut, en Acadie, une époque riche en légendes et superstitions. L'ethnologue Jean-Pierre Pichette définit la légende comme "le récit oral d'une action fantastique présentée dans une perspective historique : elle nomme les personnages, cite les lieux, indique l'époque, apporte des preuves et s'appuie sur des témoins bien identifiés. Ces circonstances exigent de l'auditeur une croyance dans les faits exposés d'autant plus grande qu'il ne peut habituellement pas les vérifier ${ }^{13}$. Ainsi, on attribue à la légende un fondement historique et un caractère local. L'ethnologue Jean-Claude Dupont remarque qu'autrefois, les légendes, qui, selon lui, sont "objets de croyance $»^{14}$, répondaient à un besoin psychologique; elles permettaient aux gens d'accepter certains phénomènes inexplicables ${ }^{15}$. Racontée comme récit véridique, la légende se propose de faire croire. Or, lié à des impressions, ce fonds de vérité historique peut être altéré par l'imagination populaire, qui s'en empare pour y ajouter des "éléments mystérieux et colorés qui dépassent et déforment la réalité ${ }^{16}$. Antonine Maillet offre dans son œuvre Chronique d'une sorcière de vent une définition qui rejoint celle des folkloristes : "Par définition, la légende se fonde sur des faits réels que le temps et l'imagination populaire déforment, amplifient et embellissent. ${ }^{17}$ Dans sa thèse doctorale, elle ajoute : "La légende, de même, grandit,

10. Antonine Maillet, Rabelais et les traditions populaires, op. cit., p. 1.

11. Antonine Maillet, Mariaagélas, Paris, Grasset et Fasquette, préface d'Yves Bergeron, 1975, 250 p. Dorénavant, les références à ce roman seront signalées au moyen de l'abréviation MAG.

12. Antonine Maillet, Pélagie-la-Charrette, Montréal, Leméac, 1979, 321 p. Dorénavant, les références à ce roman seront signalées au moyen de l'abréviation PLC.

13. Jean-Pierre Pichette, "Enquête sur les légendes de Charlesbourg », dans Nord 7 , 1977, p. 42.

14. Jean-Claude Dupont, Contes et légendes : traditions populaires acadiennes, Sainte-Foy, Éditions GID, 2002, p. 75.

15. Jean-Claude Dupont et Jacques Mathieu, Héritage de la francophonie canadienne Traditions orales, Québec, PuL, 1986, p. 71.

16. Anselme Chiasson et al., "Le folklore acadien », dans Jean Daigle (dir.), L'Acadie des Maritimes, Moncton, Université de Moncton, Chaire d'études acadiennes, 1993, p. 677.

17. Antonine Maillet, Chronique d'une sorcière de vent, Montréal, Leméac, 1999, p. 139. 
s'allonge, se développe dans un sens ou dans l'autre, selon les vents et les sucs de la vie. ${ }^{18}$ Les légendes peuvent donc contenir des exagérations et des détails faux. Toutefois, pour Dupont, "que les ancêtres y aient cru ou non a moins d'importance que la compréhension du message qu'ils cherchaient à transmettre à leur auditoire et à la postérité $»^{19}$. Véridiques ou non, certaines légendes, transmises d'une génération à l'autre, ont survécu jusqu'à nos jours. Catherine Jolicœur, dans son livre Les plus belles légendes acadiennes, présente plus d'une centaine de légendes divisées selon 18 thèmes principaux ${ }^{20}$; on trouve des références à la majorité d'entre eux dans les œuvres de Maillet. Certains thèmes sont seulement mentionnés en passant, comme la chasse-galerie, les loups-garous, les feux follets, le diable, l'Antéchrist, le septième du septième, l'arrêteux de sang, saint Antoine, les sirènes de mer et le Petit Albert ${ }^{21}$, "livre qui contenait tous les secrets de la magie $»^{22}$. Considérez, par exemple, le passage suivant, provenant du roman Mariaagélas : "Quand un chrétien vend son âme au diable, y a pu rien à son épreuve : il peut aussi ben courir la chasse-galerie comme faire son sabbat avec les sorciers, comme manger des enfants dans les messes noires. Contre les vendus du diable et les loups-garous, y a un seul remède, c'est de se tenir le plus fort possible en état de grâce » (MAG, 180-181). Ou encore, ce deuxième passage qui est axé sur la croyance à certains de ces thèmes : "les lutins, loups-garous, sorcières et feux follets... ceux-là, ne vous en faites pas, étaient bien réels en Acadie » (PLC, 113).

Avant d'aller plus loin, il est important de faire la différence entre la légende et la croyance (ou superstition). Les légendes sont généralement basées sur des croyances traditionnelles de nature superstitieuse. Selon le Petit Robert, la superstition, c'est le fait de croire que certains actes, certains signes entraînent, d'une manière occulte et automatique, des conséquences bonnes ou mauvaises. Alors, une personne qui est superstitieuse croit qu'il existe des esprits qui peuvent contrôler nos actions et nous prévenir de l'avenir. Comme l'explique la narratrice de Mariaagélas, "la superstition c'est le chat noir, l'échelle, le miroir cassé... " (MAG, 169). Ces croyances, qu'on nomme aussi présages ou avertissements, sont donc des signes qui annoncent à l'avance tel ou tel événement. Dans sa

18. Antonine Maillet, Rabelais et les traditions populaires, op. cit., p. 57.

19. Jean-Claude Dupont et Jacques Mathieu, op. cit., p. 72.

20. Catherine Jolicœur, Les plus belles légendes acadiennes, Montréal, Stanké, 1981, $280 \mathrm{p}$.

21. Pour une description de ces thèmes légendaires, voir, entre autres, Alain Doucet, $L a$ Littérature orale de la Baie Sainte-Marie, Québec, Éditions Ferland, 1965; Catherine Jolicœur, op. cit.

22. Antonine Maillet, Rabelais et les traditions populaires, op. cit., p. 88. 
thèse de doctorat, Maillet note que le présage « est une des formes les plus vivantes, en Acadie. On lit des présages partout : dans le temps, les choses, les animaux, les personnes [...] $»^{23}$. Elle ajoute : "Exceptionnellement, les signes annoncent $d u$ bonheur ou de la chance. ${ }^{24}$ Or on retrouve plutôt dans ses œuvres des références à des superstitions qui annoncent de la malchance : par exemple, pour les charretiers, une comète est signe d'un malheur à venir $(P L C, 57)$, et le grincement de roues d'une charrette invisible annonce le trépas. De même, dans Mariaagélas, on dit que les dauphins dansent la mort $\left(M_{A G}, 230\right)$ et qu'il n'y a " pas plus grand présage de malheur qu'un vivant dans un tombeau » $\left(M_{A G}, 186\right)$. En fait, tout le village eut peur quand Polyte tomba au fond d'une fosse ouverte " parce qu'on savait, dans ce pays, qu'on ne se moque pas de ce genre d'avertissements » $\left(M_{A G}, 242\right)$. Par ailleurs, Bidoche trouve une croix échouée dans "l'harbe » à outarde qui, selon les dires de sa mère, annoncerait une mort prochaine $\left(M_{A G}, 215\right)$. De plus, la venue d'une tortue géante dans la baie annonçait la mort de Ferdinand. D'après la narratrice, " on avait toujours su au pays que l'apparition soudaine et imprévue d'une bête monstrueuse ne pouvait rien apporter de bon ", et cette tortue, "vieille d'un siècle, et mangeuse de homards, s'en venait confirmer la croyance populaire » (MAG, 227). Quelques gestes ou objets religieux apportent cependant de la chance. Pélagie-la-Charrette, par exemple, grimpa dans la charrette de la Mort "le pied gauche premier pour la chance... » (PLC, 315). Et, lorsque la grand-mère, Anne-Marie-Françoise, se voit prise dans la charrette embourbée à Salem, "ses doigts s'acharnent sur les grains de son chapelet pendant qu'elle marmonne des Salue, Marie... Salue, Marie... Salue, Marie... » (PLC, 264). Mariaagélas, elle, "s'accrocha au rosaire qui lui pendait à la ceinture comme à une corde de sauvetage » pour avoir de la chance à son arrivée chez les douaniers américains (MAG, 151). Ainsi, si un signe se réalise, l'événement devient légendaire.

Les romans de Maillet présentent entre autres des lutins, c'est-à-dire ces petits êtres fantastiques qui, selon la tradition orale, fréquentaient les granges, tressaient les crinières des chevaux et les faisaient courir toute la nuit. On a connu ces lutins des granges un peu partout en Acadie, surtout au Nouveau-Brunswick; Catherine Jolicœur inclut plus d'une dizaine d'exemples dans son recueil ${ }^{25}$. Pour ce qui est de la Nouvelle-Écosse, Alain Doucet en recueille quelques versions à la Baie Sainte-Marie ${ }^{26}$ et, en 1961, Anselme Chiasson note, concernant le village de Chéticamp : « Il n'y a pas très longtemps encore, les gens croyaient dur comme fer aux

23. Id., p. 89.

24. Ibid.

25. Catherine Jolicœur, op. cit., p. 43-52.

26. Alain Doucet, op. cit., p. 45-47. 
lutins. » ${ }^{27}$ Dans Mariaagélas, le terme "lutins » est mentionné lors d'une discussion sur la « demi-forge » des Allain qui, selon la narratrice, «n'avait pas de quoi ferrer convenablement une poule » $\left(M_{A G}, 29\right)$. Ne croyant pas à l'existence de cette forge, Paul, le père de Louis «voulait plutôt croire que les chevaux qui ne passaient pas par son enclume se ferraient tout seuls ou se faisaient ferrer par les lutins... » $\left(M_{A G}, 29\right)$. La narratrice continue : "On n'allait tout de même pas mettre en doute le pouvoir des lutins. Car au dire de Paul à Gélas, les lutins capables de tresser la crinière des chevaux et de les chevaucher une nuit durant, étaient sûrement de calibre à leur mettre des fers aux sabots. » $\left(M_{A G}, 29\right)$

Ensuite, Maillet parle souvent de trésors cachés dans ses romans. Dans Pélagie-la-Charrette, on discute d'un trésor acadien : la fortune des LeBlanc. Selon la tradition orale, un dénommé Jean LeBlanc aurait « laissé un trésor caché quelque part... à tous ces descendants » (PLC, 183). Or, comme le fait remarquer un des charretiers, "[...] le dénommé Jean LeBlanc en a point laissé, de descendants »; pourtant, cela n'a pas empêché les gens de raconter la légende puisque, selon la narratrice, "tous les aïeux l'avont rapportée, celle-là » (PLC, 183). On parle aussi souvent des trésors du capitaine Kidd. En fait, d'après les études en littérature orale, on trouve des histoires sur les trésors cachés dans presque toutes les régions maritimes où l'on recevait la visite des pirates, connus pour cacher leurs fortunes. À Pubnico, en Nouvelle-Écosse (mon village d'origine), des récits sur les trésors du capitaine Kidd circulent toujours ${ }^{28}$. Né pendant la première moitié du xvII ${ }^{e}$ siècle, il fut le pirate le plus fameux de l'époque. Plusieurs ont écrit à son sujet et, selon Désiré d'Éon, " autant de fouilles ont été faites pour trouver les trésors qu'il aurait cachés le long des côtes de l'Amérique ${ }^{29}$. Mariaagélas, "rêvait au Capitaine Kidd qui, au dire des vieux, était venu enterrer ses trésors dans les sables de la baie » (MAG, 34). La narratrice note : "On jurait qu'il s'en cachait au moins deux dans l'anse et plusieurs le long de la dune. Des hommes les avaient même aperçus en creusant mais n'avaient pas réussi à les déterrer, à cause du diable. Ce maudit boiteux ${ }^{30}$ y tenait ses trésors, et jouait de toutes ses chaînes chaque fois qu'un déterreux s'approchait avec une pelle de l'une de ses cachettes »

27. Anselme Chiasson, Chéticamp : histoire et traditions acadiennes, Moncton, Éditions des Aboiteaux, 1961, p. 263-264.

28. Carmen d'Entremont, «Contes, légendes, histoires et mystifications : la tradition orale de Pubnico-Ouest », mémoire de maîtrise, Lafayette, University of Louisiana at Lafayette, 2006, p. 112-116.

29. Désiré d'Éon, Histoires de chez-nous : faits et anecdotes d'un temps qui n'est plus, Yarmouth, L'imprimerie Lesbarbot, 1977, p. 38.

30. Maillet note dans sa thèse doctorale qu'une des légendes médiévales circulant en Acadie dit que le diable a le pied bot (Rabelais et les traditions populaires, op. cit., p. 64). 
$\left(M_{A G}, 34\right)$. Il est intéressant de noter qu'à Pubnico aussi, certains pensaient que des esprits protégeaient les trésors du capitaine Kidd; selon la légende, on aurait entendu des sons de chaînes et de cloches dans ces lieux, ce qui fut attribué au diable ${ }^{31}$. En fait, selon la tradition populaire, "des forbans auraient décapité un membre de leur équipage pour l'enterrer avec le trésor dont il serait alors devenu le gardien, ou auraient voué ce trésor au diable qui devait ensuite en assurer la garde ${ }^{32}$. Pour sa part, Antonine Maillet note que "le vacarme des chaînes du diable est un vieux thème qui résonne dans maintes légendes "33. De toute façon, comme le fait remarquer la narratrice, " très peu d'hommes avaient réellement trouvé de ces trésors, mais on en parlait beaucoup... » $\left(M_{A G}, 42\right)$.

Ensuite, dans Pélagie-la-Charrette, le conteur-radoteux Bélonie-le-Vieux, qui, "pour sa part, ne rapportait que du vrai authentique », fait connaître la légende de la cloche de Grand-Pré, cloche "qu'il avait lui-même entendue sonner en haute mer, en plein ouragan, croyez-le ou pas » (PLC, 183). II raconte que "dans la baie des Chaleurs, au sud de la Gaspésie, on peut encore entendre à la veille d'une tempête sonner les cloches de Grand'Pré qu'un bateau au nom de la Tourmente était chargé jadis de transporter à Gaspé »; il explique que "le bateau aurait eu tort, apparence, de chercher à s'emparer des cloches au lieu de les mettre à l'abri, car le lendemain, il périt en mer, le bougre », et selon la tradition orale "les cloches de Grand'Pré continuent depuis ce jour à tinter dans la tempête pour avertir les pirates à venir de se tenir loin des trésors acadiens » $(P L C, 183)$. Le recueil Contes et légendes de Jean-Claude Dupont contient un récit nommé "La Cloche qui pleure » qui offre des ressemblances avec celui de Bélonie ${ }^{34}$. Cette deuxième version concerne deux hommes qui, après la Déportation, volèrent la cloche de la chapelle des ruines de Louisbourg. En route, cette cloche faillit les écraser à quelques reprises, et lorsque les hommes blasphémèrent en traversant un pont, les chevalets de soutien s'écroulèrent, entraînant l'équipage dans les eaux. Les deux malfaiteurs se noyèrent et on entend depuis des pleurs de la cloche s'élever de la rivière. Dans les deux cas, les voleurs ont été punis par une force divine. Selon Catherine Jolicœur, on trouve de nombreux exemples de cette forme de châtiment dans les anciennes mythologies et dans les traditions orales, y compris en Acadie ${ }^{35}$.

31. Carmen d'Entremont, op. cit., p. 112-114.

32. Anselme Chiasson et al., op. cit., p. 682.

33. Antonine Maillet, Rabelais et les traditions populaires, op. cit., p. 64.

34. Jean-Claude Dupont, Contes et légendes, op. cit., p. 126-127.

35. Catherine Jolicœur, op. cit., p. 151. Ce recueil contient un chapitre sur les punitions et la justice immanente, p. 151-157. 
D’autres légendes occupent une plus grande place chez Maillet, comme la célèbre légende du vaisseau fantôme, née d'un phénomène naturel encore inexpliqué; elle met en scène un grand navire à voile mystérieux qui se promène à l'horizon en feu. La forme du navire varie d'une version à l'autre, mais il est presque toujours en feu et, d'après Anselme Chiasson, il est parfois peuplé de marins qui courent sur le pont et grimpent dans les haubans ${ }^{36}$. Dans Pélagie-la-Charrette, Bélonie, qui croyait avoir laissé les bateaux fantômes en Acadie, voit ce vaisseau quand les charretons arrivent enfin à la mer après avoir traversé Charleston. Fixant l'horizon, il aperçoit ce " mauvais signe » et crie de peur : «le vaisseau fantôme! » À ce moment, la narratrice déclare : "Le vaisseau fantôme, on le connaît. Depuis des siècles qu'on se passe ce bâtiment maudit, brûlant en mer, tantôt au nord, tantôt à l'est, expiant éternellement une faute perdue dans la nuit des temps mais que le ciel et la terre refusent de pardonner. Et brûle et rebrûle, jusqu'à la fin du monde... » (PLC, 84). Elle décrit ensuite la scène : "Les charrettes frémissent devant ce brasier vivant qui lance des éclairs dans le ciel en fonçant sur la barre du jour. Un bâtiment en feu, une voilure en feu, des mousses, des matelots, un quartier-maître flambant au soleil... on aperçoit... clairement l'équipage fantôme sur le pont, les mains noires de cendre et le visage allumé par les dernières flammes mourantes... » Bélonie suggère : "Le quatre-mâts anglais qui a péri en mer corps et biens, lors du Grand Dérangement » (PLC, 85). Dans la tradition orale, on donne de multiples explications pour cette apparition, mais la plupart du temps, on dit que c'est le navire de pirates qui aurait été condamné à brûler pour ces crimes. Dans Mariaagélas, lorsqu'un matelot aperçoit « un navire en feu à l'horizon », le capitaine des îles Saint-Pierre-et-Miquelon évoque avec son équipage la légende "de ce navire qui brûle depuis des siècles sur les côtes de l'Atlantique ": "C'était un châtiment éternel. L'équipage de ce bateau s'était rendu coupable de quelque faute grave: le viol d'une jeune Indienne, ou le massacre d'un village, ou l'incendie des maisons de Grand-Pré... on ne savait plus très bien. Il circulait plusieurs versions sur la nature du péché que devait expier ce bateau-là. Tout ce dont on était sûr, c'est qu'il expiait. Depuis des générations. Le châtiment s'était abattu sur le navire dès le lendemain de son forfait : il avait péri corps et biens dans une tempête en haute mer. Depuis ce jour-là, il réapparaissait périodiquement et brûlait de nouveau. Mais ce n'était plus le bateau qui brûlait, c'était son fantôme. » $\left(M_{A G}, 192\right)$.

Catherine Jolicœur, qui a recueilli le plus grand nombre de légendes en Acadie, a fait sa thèse de doctorat sur ce vaisseau fantôme; elle aurait

36. Anselme Chiasson et al., op. cit., p. 680. 
recueilli plus de 600 versions différentes de la légende ${ }^{37}$. Pour Anselme Chiasson, c'est la légende acadienne la plus célèbre ${ }^{38}$. On trouve des variantes un peu partout en Acadie, mais, selon Dupont, ce navire est apparu le plus souvent dans la baie des Chaleurs, au Nouveau-Brunswick ${ }^{39}$. Dans ce milieu, où des centaines d'individus affirment avoir vu le bateau fantôme, on nomme cette apparition "Feu du mauvais temps », car le passage de ce bâtiment pouvait annoncer une tempête $4^{0}$. Dans Mariaagélas, le capitaine des îles Saint-Pierre-et-Miquelon affirme cette croyance : "le bateau-fantôme apparaît la veille d'une tempête. C'est pour ça qu'on l'appelle aussi le feu du mauvais temps ». Et la croyance se transformait en récit légendaire car "le lendemain, la tempête éclata» $\left(M_{A G}, 192\right)$. On donne cette même explication dans Pélagie-la-Charrette : "Le malheur de ce bâtiment forban, gouverné par le diable en personne, sera de voguer entre la vie et la mort jusqu'à expiation de son forfait, incommensurable. Et chacune de ses apparitions sera suivie le lendemain d'une tempête en mer: c'est pourquoi le bateau fantôme porte aussi le nom de feu du mauvais temps. » (PLC, 85).

Il y a une autre légende qui joue un rôle important dans l'œuvre de Maillet : celle de la charrette de la mort, c'est-à-dire cette charrette invisible, qu'on entend approcher de loin et qu'on nomme aussi "charrette fantôme » ou "Faucheuse ». Ronald Labelle note que Maillet avait recueilli, avant la parution de son recueil en 1971, des versions de cette légende à Caraquet, ainsi qu'aux Îles-de-la-Madeleine ${ }^{41}$. Toutefois, lorsqu'il la questionna au sujet de son intérêt pour celle-ci, elle expliqua qu'elle avait d'abord été frappée par une image venue d'un film de Carl Theodore Dreyer ${ }^{42}$. Pour ce qui est de ses romans, on en parle surtout dans Pélagie-la-Charrette, où cette charrette légendaire joue un rôle central dans le récit43. Dès le début, Bélonie-le-Vieux, "prophète de malheur » $(P L C, 22)$, dresse en face de la charrette de la vie, mise en marche par Pélagie, la charrette de la mort qui, selon Sheila Henderson, « rappellera continuellement la menace qui plane sur les personnages acadiens du

37. Pour une étude comparative du vaisseau-fantôme, voir Catherine Jolicœur, Le Vaisseau fantôme : légende étiologique, Québec, PuL, 1970, 337 p.

38. Anselme Chiasson et al., op. cit., p. 680.

39. Jean-Claude Dupont, Contes et légendes, op. cit., p. 108.

40. Anselme Chiasson et al., op. cit., p. 681.

41. Ronald Labelle, « Une charrette qui a traversé l'océan? Les reflets de l'Ankou breton en Acadie », dans Jean-Pierre Pichette (dir.), Port Acadie, n 13-14-15 (" La Résistance des marges »), Université Sainte-Anne, Pointe-de-l’Église, 2008-2009, p. 285.

42. Id., p. 287.

43. Voir l'article de Sheila Henderson. 
retour ${ }^{44}$. À travers tout le texte, on trouve des références à cette légende, surtout par l'entremise de Bélonie qui, lui, "l'avait vue de près tant de fois, entendue, Bélonie, entendue, car personne jamais n'avait vu ce sombre fourgon... une charrette qui parcourait le monde depuis le commencement des temps» $(P L C, 17)$.

Comme c'est le cas pour la majorité des légendes, les récits sur la charrette fantôme varient. Or, dans la plupart des cas, on parle du "flacottage » ou du grincement de ses roues, comme c'est le cas dans Pélagie-la-Charrette : "[...] dans les propres ornières de la charrette de Pélagie grinçaient les roues invisibles de la charrette de la Mort » (PLC, 21). La narratrice du roman explique : "la charrette grinçait des roues pour avertir les vivants de s'enlever de son chemin, qu'elle ne venait que pour les trépassés, les moribonds ou les marqués du destin » $(P L C, 22)$. Jean-Claude Dupont confirme cette croyance dans ses Contes et légendes : "on prétendait que lorsque la charrette de la mort apparaissait à un homme, une personne de sa famille allait mourir durant l'année ${ }^{45}$. Justement, lors du grand voyage de retour, on entend surtout les roues de cette charrette invisible à chaque menace de mort : "C'est ainsi qu'on l'avait entendue à la mort du défunt Sirois à Basile Gautreau... et la veille du trépas de la pauvre Barbe à Babée... et lors du naufrage de la défunte Espérance... » $(P L C, 21)$. Marie Cormière entend aussi la charrette de la mort lorsque son fils, le petit Frédéric, tombe gravement malade : "Arrêtez, arrêtez ce bruit, ces roues de charrette qui grincent, arrêtez-les, l'enfant est malade... j'entends les essieux, le fouet... arrêtez-les... » $(P L C, 55)$ Personne ne réussit à l'arrêter et l'enfant meurt. Ensuite, lorsque la charrette de Pélagie et les bœufs s'embourbent dans les marais de Salem, on se débat dans la vase mouvante. À ce moment, Bélonie-le-Vieux, qui prend conscience qu'elle " est là la Faucheuse " $(P L C, 269)$, " reçoit en pleine face le grincement de ses roues et le sifflement du fouet qui fend l'air » (PLC, 270). Et, selon les témoignages, quand Bélonie lui-même mourut à l'automne, on entendit la nuit de son décès "le grincement d'une charrette au loin » (PLC, 289). Enfin, Pélagie, avant de mourir, fut la dernière à entendre le grincement de la "Faucheuse » : "Bélonie... je t'entends... Je reconnais le grincement des roues et le sifflement du fouet qui fend l'air... » (PLC, 314-315).

Il y a un autre thème qui semble récurrent chez Maillet : celui des revenants, qui, selon Dupont, "étaient souvent des personnes décédées qui séjournaient au purgatoire en attendant d'entrer au ciel $»^{46}$. Anselme Chiasson note que « les apparitions de défunts sont une source de légendes

44. Id., p. 91.

45. Jean-Claude Dupont, Contes et légendes, op. cit., p. 119.

46. Id., p. 115 . 
innombrables et variées dans toutes les régions acadiennes ${ }^{47}$. Autrefois, on pensait que les morts revenaient sur terre pour transmettre des messages, accomplir des tâches inachevées, rendre un objet emprunté, régler des dettes et demander des prières ${ }^{48}$. Alors, lorsqu'on entendait un bruit mystérieux ou qu'on apercevait une apparition inexplicable, on pouvait facilement s'imaginer que c'était un revenant. Effrayés par ces phénomènes, les gens récitaient souvent des prières ou faisaient dire des messes pour les défunts, en espérant qu'ils ne reviendraient plus. Selon la croyance populaire, "celui qui meurt sans avoir tenu une promesse faite à quelqu'un devient une âme en peine ${ }^{49}$. De plus, les esprits des gens qui étaient morts sans funérailles revenaient demander des prières pour le repos de leur âme ${ }^{50}$. La veuve à Calixte, par exemple, "hurlait à qui pouvait l'entendre que la femme du vieux Louis à Paul à Gélas avait enterré son homme sans même l'ensevelir, et que l'âme du vieux pouvait bien encore errer entre ciel et terre par les nuits qui vont » $\left(M_{A G}, 55\right)$.

On craignait surtout l'apparition des morts le soir de la Toussaint ( $1^{\text {er }}$ novembre) et le jour des Morts ( 2 novembre) ${ }^{51}$; comme l'explique la narratrice dans Mariaagélas : "[...] c'était la tradition chez les morts de revenir piquer le nez aux alentours de la Toussaint. On avait beau allumer toutes les chandelles bénites à Pâques, et pavoiser les fenêtres de rameaux, et s'abstenir de bêcher ou de labourer au Jour des Morts ${ }^{52}$... non! Ça ne servait à rien... » $\left(M_{A G}, 55\right)$. Mariaagélas avait averti le superstitieux Soldat-Bidoche, dont le père, mort de la grippe espagnole, avait été enterré "sans sarémonie » $\left(M_{A G}, 50\right):$ : [...] je me méfierais, moi, de ces morts mal enterrés, quand c'est que viendra la Toussaint. Y en vient tous les ans, c'te jour-là, de ces effarés du pigatouère, avec leux jambes molles et leu face pâle... Si tu les appelles, c'est sûr qu'ils viendront » (MAG, 50-51). Les avertissements de Mariaagélas se firent entendre à travers le village " et en moins de trois jours, la paroisse avait à demi ressuscité une bonne douzaine de ses plus récents morts et prévoyait les pires catastrophes pour la Toussaint » (MAG, 55).

À la Toussaint, les jeunes allaient faire peur aux habitants la nuit, par exemple en suspendant des bonshommes fabriqués en guenilles aux

47. Anselme Chiasson et al., op. cit., p. 680.

48. Voir Catherine Jolicœur, op. cit., p. 95-119.

49. Id., p. 96.

50. Gary Butler, Histoire et traditions orales des Franco-Acadiens de Terre-Neuve, Sillery (Québec), Éditions du Septentrion, 1995, p. 107.

51. Plusieurs croyances sur les morts entouraient les $1^{\text {er }}$ et 2 novembre; voir Jean-Claude Dupont et Jacques Mathieu, op. cit., p. 53-56.

52. Dans sa thèse doctorale, Antonine Maillet explique qu'il est interdit de bêcher la terre le jour des Morts pour ne pas déranger l'éternel sommeil de ceux qui y sont enfouis, Rabelais et les traditions populaires, op. cit., p. 74. 
arbres devant les maisons ${ }^{53}$. Enfin, ce sont les croyances entourant les $1^{\text {er }}$ et 2 novembre qui donnèrent naissance au revenant du Lac à Mélasse qu'on retrouve dans le roman Mariaagélas. Cette légende naquit à la suite des avertissements de Maria, lorsque Soldat-Bidoche arriva à la forge des Allain en bégayant : "C'est blanc... ça grouille... je l'ai vu... au boute du Chemin des Amoureux. Je crois que c'est mon défunt père » (MAG, 60). Selon la narratrice, "tout ça se passait le soir de la Toussaint qui, dans le calendrier de l'époque, se trouvait la nuit qui précédait le Jour des Morts ». Elle ajoute : "Or personne en ce temps-là n'aurait confondu le Jour des Morts et le soir des tours, les tours se jouant entre vivants ${ }^{54}$. Ensuite, elle confirme l'apparition du revenant : "[...] il était là, le revenant, tout gaillard et tout vivant au bout du Chemin des Amoureux. Il gigotait, se trémoussait... " (MAG, 61). Bidoche, qui "avait toujours eu peur des morts... peur de la nuit, des éloèzes, et du feu chalin... était persuadé que le revenant était revenu tout exprès pour lui, au Chemin des Amoureux, et que ça ne pouvait pas être autre chose que son père ». En plus d'être enterré sans cérémonie, "il était parti trop vite, le défunt, avant d'avoir eu le temps de régler ses comptes et de mettre ordre à ses affaires " (MAG, 65). La Veuveà-Calixte suggéra : "me r'semble qu'un défunt qui se donne le trouble de revenir sus la terre au boute de trois ans, il doit vous aouère un patchet de remords à l'embouchure de la conscience... » Ainsi, Bidoche pensait qu'il était revenu pour la corde de bois qu'il avait abandonnée ou la doré (petite embarcation à fond plat servant principalement à la pêche) qu'il n'aurait pas rendue au p'tit Georges (MAG, 66).

Après avoir disparu pendant trois jours, ce revenant réapparaissait au Lac à Mélasse : "du jour au lendemain, il avait charrié toute sa foule de curieux au Lac à Mélasse... » (MAG, 74). Toute la paroisse se rendait là les nuits pour voir cette apparition. Mais Mariaagélas, elle, "en riait tout seule dans son lit » $\left(M_{A G}, 73\right)$. Et la veuve à Calixte qui, "à elle seule... valait un service d'espionnage ", comprit que "le revenant était une couverture » $\left(M_{A G}, 80\right)$. Elle découvrit que c'était la fabrication de Mariaagélas : "Un drap, figurez-vous, un drap noir d'un bord et blanc de l'autre, qui tournaillait sur une poulie! Heh! pas si sotte la fille à Gélas, faudrait s'en rappeler. Et dire que ç'avait fait courir toutes les paroisses et jusqu'aux concessions, cette machine-là. » $\left(M_{A G}, 85\right)$ Enfin, la légende, démystifiée, se transformait en anti-légende ${ }^{55}$ ou anecdote. À cause des croyances religieuses, on attribuait plusieurs phénomènes mystérieux

53. Jean-Claude Dupont, Contes et légendes, op. cit., p. 116.

54. Ce passage qui met en lien le jour des Morts avec le soir des tours annonce en fait la suite de l'histoire du revenant du Lac à Mélasse.

55. On nomme aussi les narrations où l'on tourne en dérision la croyance aux esprits des vieux d'auparavant « anti-légende » (Gary Butler, op. cit., p. 113). 
aux revenants. Or, comme ce fut le cas avec le revenant du Lac à Mélasse, on trouvait parfois plus tard une explication valide. Le bateau fantôme de Bélonie, par exemple, se transforme en bateau plein d'Acadiens vivants $\left(M_{A G}, 88\right)$. À Pubnico-Ouest, une jeune fille crut recevoir la visite d'une tante décédée lorsqu'elle entendit un bruit étrange. Supposant que l'esprit avait besoin de prières, elle s'était mise aussitôt à prier. Enfin, elle sortit de sa chambre et découvrit que les bruits mystérieux provenaient de la chambre voisine, où sa sœur et des amis ouvraient des pastilles de menthe enveloppées de plastique ${ }^{56}$.

Comme c'est le cas pour la plupart des récits présentés chez Maillet, cette légende serait basée sur des faits réels. Selon Ronald Labelle, Maillet se serait inspirée de la légende du revenant de Richibouctou, qui circulait à l'époque dans ce milieu57. Dans une enquête menée par l'ethnologue Jean-Pierre Pichette, la conteuse Séraphie Daigle-Martin, Acadienne de Richibouctou, discute du revenant en question. Selon elle, c'était un " dummy qu'ils (les contrebandiers) aviont fait faire avec des broches pour pouvoir traverser avec de la boisson... sans ramasser la police ${ }^{58}$. Elle continue en expliquant que cela avait piqué la curiosité des villageois, qui pensaient que c'était un revenant, ce qui permettait aux vendeurs de boissons de faire leur commerce sans attirer l'attention. Un dénommé Arthur Daigle de Pointe-Sapin aurait même écrit une chanson pour commémorer cet esprit; le refrain se chante comme suit : "Tout le monde en parle, parle, parle, parle du revenant de Richibouctou. 59

Les romans d'Antonine Maillet font aussi connaître la médecine populaire acadienne ${ }^{60}$. En faisant la lecture de Pélagie-la-Charrette, par exemple, on apprend qu'autrefois, on réglait les boyaux [amélioration du transit intestinal] à l'harbe-à-dindon (PLC, 43); on se réanimait les joues au jus de bettes (PLC, 136); on s'attaquait aux fièvres à coups de hareng salé et de rondelles d'oignons sous la plante des pieds, et à coups de graines de lin pour faire crever les abcès $(P L C, 242)$. Ronald Labelle divise

56. Carmen d'Entremont, op. cit., p. 145-147.

57. Cette information fut notée lors d'une discussion avec M. Labelle le 29 novembre 2010.

58. Centre acadien, Université Sainte-Anne, coll. Jean-Pierre Pichette, enreg. $n^{\circ} 3731$.

59. Ibid.

6o. Pour une étude de la médecine populaire en Acadie, voir entre autres Ronald Labelle, «La médecine populaire en Acadie - les sources d'un savoir traditionnel », dans Entre Beauce et Acadie - Facettes d'un parcours ethnologique, textes réunis par Jean-Pierre Pichette et al., Québec, Presses de l'Université de Laval, 2001, p. 321-329; Ronald Labelle, "Éléments naturels et surnaturels dans la médecine populaire acadienne », dans Ronald Labelle et Lauraine Léger (dir.), En r'montant la tradition, Moncton, Éditions d'Acadie, 1982, p. 128-148. 
la médecine populaire en trois types de traitements ${ }^{61}$. Premièrement, il y a les remèdes à base de substances naturelles, qui étaient généralement préparés à l'intérieur des familles, comme ceux qui précèdent. Ensuite, il y a la médecine surnaturelle, qui se divise en deux domaines distincts. En fait, c'est cette branche qui intéresse ici puisque, par son côté magicoreligieux, elle est liée aux croyances et à la légende. D'abord, il y a les traitements (ou rituels) qui font appel à la foi religieuse ou aux croyances superstitieuses. Par exemple, d'après Célina, la guérisseuse du roman Pélagie-la-Charette, on se chaussait du pied gauche pour se garder du mal de dent; selon elle, "c'était croyance et coutume » (PLC, 135). En l'absence du prêtre, des gens ordinaires se servaient également de prières et d'objets religieux, comme les médailles scapulaires, les croix ou l'eau bénite, pour guérir. Et lorsque Pélagie tomba gravement malade, on la lava dans "l'eau de source cueillie avant l'aube au matin de Pâques " (PLC, 307) et, au printemps, on attendait la tombée de "la neige miraculeuse de mai qui guérit tous les maux » (PLC, 308). Selon la croyance, l'eau de Pâques, que l'on puise à contre-courant, aurait, comme l'eau (ou la neige) de mai, "la vertu de guérir certaines maladies... " ${ }^{62}$ Ensuite, le crucifix des Allain, qui avait servi à "éclairer la route et chasser les démons " (PLC, 154), et les prières du vieux Jean-Baptiste jouèrent un rôle dans la guérison de la petite Virginie. Lorsqu'on alla chercher Jean-Baptiste pour réciter les prières, sa réaction initiale fut : " Je suis point un prêtre. " Mais, quand il eut fini ses prières, Virginie ouvrit miraculeusement les yeux et sa fièvre s'éteignit $(P L C, 298)$. On trouve en fait des récits semblables dans la majorité des milieux acadiens. Après avoir perdu plusieurs bébés, une femme de Pubnico-Ouest demanda à ses amies de faire une neuvaine lorsqu'elle tomba encore enceinte. Et cette fois, elle ne perdit pas son bébé; selon elle, la neuvaine avait fonctionné ${ }^{63}$. De même, à la Baie Sainte-Marie, une femme fut guérie d'une plaie sur la jambe en faisant une neuvaine ${ }^{64}$.

Pour finir, il y a les traitements à caractère magique. À l'époque, on retrouvait dans chaque région acadienne des guérisseurs aux pouvoirs mystérieux. Il y avait, entre autres, le charmeur de mal de dents, le « passeux de verrues ", qui pouvaient faire disparaître un mal en posant un geste ou en récitant une formule secrète, et l'« arrêteux de sang », celui qui pouvait arrêter le saignement d'une blessure ou encore un saignement

61. Ronald Labelle, "La médecine populaire en Acadie », op. cit., p. 129. Voir aussi Gary Butler, op. cit., p. 77-93.

62. Jean-Claude Dupont, Le Rituel de l'année - Traditions populaires acadiennes, Sainte-Foy, Éditions GID, 2002, p. 75.

63. Carmen d'Entremont, op. cit., p. 135-137.

64. Centre acadien, Université Sainte-Anne, coll. Jean-Pierre Pichette, enreg. $n^{\circ} 3731$. 
de nez sans même toucher à la personne affligée ${ }^{65}$. Ceux-ci recevaient certains dons par l'intermédiaire d'un parent ou naturellement, en étant le septième fils ou la septième fille d'une famille. On trouve chez Maillet quelques-uns d'entre eux. Comme le signale la narratrice de Mariaagélas : "Guérisseuses, rabouteux, arrêteux de sang, vendeurs de sirops et d'onguents Ralley, tous ces êtres sortis des almanachs faisaient partie du paysage des côtes comme la mer et les herbes salines » (MAG,179). Dans ce roman, on explique qu'on allait " qu'ri' un docteur... pour l'enflammation des boyaux, les poumons au vif, pis les rhumatiques enflammatoires; ben pas pour arrêter le sang ou pour ramancher les ous cassés » $\left(M_{A G}, 177\right)$. Antoine à Zéphir, par exemple, "ramanchait tous les os cassés ou déboîtés le long des côtes » $\left(M_{A G}, 179\right)$. Tit-Lou à Magloire, qui s'était enfargé dans un "nique d'ours qui dorm[ai]ent encore » et qu'on avait "trouvé déchiré à coups de griffes de l'échine au cagouette " avait eu besoin des services du ramancheux; on dit que "ç'a pris des mois au ramancheux pour le raccommoder » $\left(M_{A G}, 144\right)$. De même, la Veuve-à-Calixte, estropiée après avoir dégringolé en bas du cap, avait aussi eu recours aux services du ramancheux; selon les témoignages, " on lui chercha un bon raboteux qui lui rabouterait son bras, un ramancheux» (MAG, 176-177). En fait, la Veuve avait aussi cherché les services d'un arracheux de dents après son accident $\left(M_{A G}, 178\right)$. Et, lors du grand voyage de retour, lorsque le charretier, Pacifique Bourgeois, "sentait un abcès lui dévorer la joue... » (PLC, 130), on voulait lui trouver un arracheux de dents. Pour sa part, Célina, en plus de s'y connaître en médecine naturelle, avait plusieurs dons, ce qui explique qu'on la nomme "sage-femme-guérisseuse-raboteuse-etdésormais-brûleuse-de-dents » (PLC, 136).

\section{Les autres genres}

En plus des légendes et croyances, les œuvres de Maillet font connaître plusieurs autres genres appartenant à la littérature orale, comme les chansons, les contes, les menteries et les dits populaires. Dans certaines cas, on ne donne que le titre de chansons du pays, comme "Malbrouc s'en va-t-en guerre " $\left(M_{A G}, 140\right)$, "L'Ave Maris Stella » et "Partons la mer est belle » $\left(M_{A G}, 185\right)$. Parmi les chansons les plus citées, il y a l'«Alouette »: "alouette, gentille alouette, alouette, je te plumerai » (PLC, 42, 229 et 278). Et la chanson "Le pommier doux », dont on trouve une version dans les Chansons d'Acadie des pères Daniel Boudreau et Anselme Chiasson, est chantée à plusieurs reprises dans Pélagie-la-Charrette par les charretiers. Ils la chantent quand ils rejoignent enfin la mer ( $P L C, 83)$, pour fêter l'arrivée de la Grand'Goule ( $P L C, 88)$, pour fêter la noce de Madeleine

65. Gary Butler, op. cit., p. 137. 
(PLC, 229 et 309) et pour fêter la Saint-Jean : «Etj'ai du grain de mil, et j'ai du grain de paille, et j'ai de l'oranger, et j'ai du tri, j'ai du tricoli, et j'ai des allumettes, et j'ai des ananas, j'ai de beaux, j'ai de beaux, j'ai de beaux oiseaux... » $(P L C, 52)$. En Acadie, cette chanson est redevenue populaire quand Édith Butler l'a ajoutée à son répertoire.

Il y a aussi dans les textes de Maillet de nombreuses allusions aux contes traditionnels. Certains d'entre eux, comme celui de "Cendrillon », sont mentionnés en passant. Deux contes, racontés par Bélonie, le radoteux-conteux-chroniqueur, occupent cependant une place plus grande. Voulant divertir les charretiers à Charleston, Pélagie propose à Bélonie: "Contez-nous l'un de vos contes joyeux» $(P L C, 72)$. Et il raconta le fameux conte de la baleine blanche ${ }^{66}(P L C, 73)$. Ce récit raconte l'histoire d'une poule qui avala son anneau d'or et qui fut mangée par un renard, lequel fut mangé par un ours, qui aboutit dans le ventre d'une baleine. Selon Évelyn Voldeng, ce conte, qui «ne semble pas être la variante d'un conte-type particulier, appartient cependant au cycle des contes de la quête où l'objet recherché est chaque fois dans un contenant plus grand ${ }^{67}$. Ensuite, un dernier jour d'automne, quand Bélonie se décida à conter une dernière fois, Pélagie avisa la troupe : "Venez, venez tous, le radoteux va conter ». Cette fois, il relata le récit d'un héros qui portait le nom de Tit-Jean Quatorze, "à cause des quatorze tours qu'il avait déjà joués à son père... » $(P L C, 282)$, et qui, égaré à travers des parois en labyrinthe, cherchait à en sortir. Ce conte, qui est, comme le note Voldeng, " originaire du vieux pays ", appartient au conte-type 650, intitulé « Jean le Fort » ou "Quatorze », où il est le plus souvent question des exploits d'un géant de force herculéenne ${ }^{68}$. Dans Mariaagélas, on parle plutôt des veillées de contes, par exemple, chez le grand-père à Boy à Polyte à Jude, et des conteurs tels "le dénommé Jude qui racontait si bien des histoires qu'on finissait par croire qu'elles lui étaient arrivées personnellement » $\left(M_{A G}, 147\right)$. On discute aussi des formules de fin des contes, qui ramènent souvent à la réalité; la narratrice donne l'exemple suivant: "Et pis y a eu un grand banquet pour fêter la princesse, et tout le monde était invité, à part de moi; ils m'avont point aparçue dans le coin du château qui passait par là et je m'en ai venu droite icitte vous conter l'histoire » (MAG, 148).

66. Voir l'article de Jacques Chaurand, "Le conte de la baleine blanche tiré de Pélagie-la-charrette d’Antonine Maillet », dans Présence francophones, n³1, 1987, p. 121-128.

67. Évelyn Voldeng, "Pélagie-la-Charrette et le folklore de la France de l'ouest », dans Raoul Boudreau, Anne-Marie Robichaud, Zénon Chiasson et Pierre M. Guérin (dir.), Mélanges Marguerite Maillet, Moncton, Éditions d'Acadie, Chaire d'études acadiennes, 1996, p. 514.

68. Id., p. 515 . 
Parmi les grandes catégories de types, il y a les contes facétieux ou contes à rires. Cette catégorie, qui contient surtout des farces sur des hommes et des femmes sots ou stupides, inclut des contes de menteurs. En fait, puisque la tradition de la menterie a été très populaire et florissante et qu'elle est fascinante et unique, plusieurs chercheurs ont classé ces récits dans une section à part. Barry Ancelet, par exemple, inclut plus d'une douzaine de ces récits parmi ses Cajun and Creole Folktales, dans une catégorie qu'il nomme "Menteries et contes forts ${ }^{69}$. Les menteries prennent la forme d'anecdotes exagérées; ce sont de fabuleuses histoires inventées, mais racontées sous la forme d'un récit authentique. Selon Ronald Labelle, "ces récits concernaient souvent des personnages réels, mais il y a toujours un élément irréel ou une exagération au centre de la narration ${ }^{70}$. Il ajoute : "Les "conteurs de menteries" essayaient de rendre leurs récits le plus invraisemblable possible, tout en les faisant croire aux auditeurs. ${ }^{71} \mathrm{En}$ Louisiane, on dit qu'il y a une différence entre la menterie (tall tale) et le mensonge (lie), la menterie étant une farce racontée pour amuser, et le mensonge, une farce racontée dans le but de tromper ou de tourmenter quelqu'un ${ }^{72}$. Selon Ancelet, les contes d'exagération sont souvent basés sur des activités familières telles que la ferme, la pêche et la chasse ${ }^{73}$. On dit que les menteurs habiles sont capables d'ajouter de nouveaux éléments sur place ou, encore, de conter des menteries improvisées ${ }^{74}$. Dans les œuvres de Maillet, les menteries se présentent quelquefois spontanément, sans avertissement. Dans Pélagie-la-Charrette, par exemple, Jean à Maxime à Maxime fit serment d'avoir vu de ses yeux monter un glaçon droit devant lui un jour de février qu'il avait pissé à 55 sous zéro $(P L C, 295)$. On dit en fait que les meilleures menteries ressortent quand deux menteurs essayent, de façon compétitive, de se devancer ${ }^{75}$. Dans Mariaagélas, deux pêcheurs participent à cette tradition des concours de menteries: "Chus nous, dit l'un, i'faisait assez frette à matin que le thermomètre a cassé et pis le zéro s'a sauvé; $y$ a un houme qui l'a vu dans le boute du Portage... Chus nous, dit l'autre, i'faisait pus frette que ça, ben j'avons pas pu le raconter à cause que les paroles nous gèliont dans la goule » $\left(M_{A G}, 120\right)$.

69. Barry-Jean Ancelet, Cajun and Creole Folktales: The French oral tradition of South Louisiana, Jackson, University Press of Mississippi, 1994, p. 115-129.

70. Ronald Labelle, Au Village-du-Bois : Mémoires d'une communauté acadienne, Moncton, Université de Moncton, Centre d'études acadiennes, 1985, p. 203.

71. Ibid.

72. Barry-Jean Ancelet, op. cit., p. xxxvı.

73. Id., p. xxxvII.

74. Ronald Labelle, Au Village-du-Bois, op. cit., p. 203.

75. Voir Barry-Jean Ancelet, op. cit., p. 116-117. 
Du point de vue de la forme, on divise la littérature orale en deux branches : la littérature mouvante, qui comprend des récits qui éprouvent des modifications selon le temps, la région et le conteur, comme les contes, les légendes et les chansons, et la littérature fixée, qui englobe des formules qui sont fixées dès les débuts et ne sont presque pas modifiées, comme les comparaisons, les surnoms et les jurons. On nomme ces genres mineurs les « dires $»^{76}$. En ce qui concerne les romans de Maillet, on trouve un peu de tout : des mots prononcés "en langue du pays" (PLC, 295), tels que " canisteaux» (bottes d'Amérindiens), "forlaque » (fille aux mœurs légères), "bessons » (jumeaux), " mocauques » (terrain marécageux), "paindoux » (gâteau), " dédjeuler » (vomir), " bagueuleux » (bavard) et "machecouèche » (raton laveur); des comparaisons comme " sourd comme un pot» (MAG,135), des jurons comme " godêche de hell »; de multiples expressions, y compris faire les choses "par les petits" $\left(M_{A G}, 236\right)$, en savoir " ni le long ni le court» $\left(M_{A G}, 74\right)$ ou " que le diable emporte » $(P L C, 176)$; et plusieurs surnoms, entre autres Pissevite, la boiteuse, le Fou, Pierre Crochu, la Catoune, Louis-le-Drôle, Boy et Thomas Picoté. En fait, Pélagie-la-Gribouille note : «Un surnom, tout le monde en $a$ » $(P L C, 143)$. Puisque plusieurs personnes avaient le même nom de famille et, dans certains cas, le même prénom, on inventait des sobriquets pour identifier les individus. En outre, on nommait rarement une personne sans ajouter le nom de son père et, parfois, de son grand-père : "Polyte à Jude au petit Bossu ». Même si les surnoms présentés dans les romans de Maillet sont parfois inventés, ils témoignent d'une pratique commune à plusieurs régions acadiennes. Anselme Chiasson note qu'à Chéticamp, "les sobriquets pullulent par centaines "77 et, d'après Ronald Labelle, ils sont aussi communs au Village-du-Bois ${ }^{78}$. Dans certains cas, des surnoms, surtout ceux qui ont une origine drôle, ont même fait l'objet d'anecdotes humoristiques. Barry Ancelet présente une farce dans son article "The Cajun Who Went to Harvard" qui met en jeu l'ignorance d'une enseignante envers cette tradition des surnoms ${ }^{79}$. L'enseignante demanda le nom de ses élèves et lorsqu'elle arriva à un jeune Cadien, il dit : «Poo Poo ». Elle lui dit qu'elle ne tolérerait pas ce genre de comportement et lui demande une deuxième fois. Il dit une nouvelle fois «Poo $P o o$ », alors elle le renvoie de la salle de classe. En sortant, le Cadien dit à son cousin : "Come on, Ca Ca, she won't believe you either ».

76. Jean-Pierre Pichette, ETHN 1013 : Tradition orale, folklore et ethnologie, manuel de cours, Université Sainte-Anne, Pointe-de-l’Église, 2006, ch. 5, p. 22.

77. Anselme Chiasson, op. cit., p. 252.

78. Ronald Labelle, Au Village-du-Bois, op. cit., p. 176-178.

79. Barry-Jean Ancelet, «The Cajun Who Went to Harvard: Identity in the Oral Tradition of South Louisiana », dans Journal of Popular Culture, vol. 23, n¹, 1989, p. 106. 
Pour conclure, les références à la culture populaire sont loin d'épuiser le folklore mis en valeur dans les romans d'Antonine Maillet. Quoi qu'il en soit, les exemples retenus illustrent bien la présence de ces récits populaires acadiens dans ses œuvres. Même si Maillet, qui est écrivaine avant tout, s'empare des récits oraux, les transformant à sa façon avant de les faire renaître dans ses romans, il est évident qu'ils s'inspirent de faits réels. On n’a qu'à se rappeler du revenant du Lac à Mélasse, transposé à partir de la tradition orale de Richibouctou. Parmi les légendes présentées, plusieurs sont universelles, comme les histoires de saints et de diable ${ }^{80}$. D'après la classification des Contes et légendes de Jean-Claude Dupont, quelques-unes sont d'origine européenne, comme les lutins, les trésors cachés et le bateau fantôme. En fait, dans sa thèse doctorale, Maillet note : "plusieurs légendes de la France médiévale nous sont parvenues intactes ou légèrement modifiées ${ }^{81}$. D'autres, comme les revenants, et les légendes historiques, comme la légende de la cloche de Grand-Pré, sont plutôt nées d'accidents ou de faits typiquement acadiens $^{82}$. Il est intéressant de noter que certains récits légendaires que se racontent les personnages mailletiens sont aujourd'hui presque complétement absents de la tradition orale acadienne. De nos jours, nous sommes presque tous scolarisés, et il est plus facile de trouver des explications scientifiques pour les légendes, ce qui justifie leur rareté. Ainsi, en plus de faire découvrir la richesse du folklore acadien, les romans de Maillet communiquent aux lecteurs des connaissances du passé et révèlent les croyances anciennes.

Les romans mailletiens, comme les récits oraux, ne respectent pas nécessairement les faits; ils sont souvent accompagnés de détails faux, exagérés ou embellis par l'imaginaire à un point tel qu'il est impossible de savoir quels détails sont authentiques. Pour Maillet, capter l'attention de ses lecteurs est tout aussi important que la transmission des faits. Néanmoins, selon Montell, "whether folklore is true or false is immaterial ${ }^{83}$; pour lui, la vérité se trouve plutôt dans les valeurs, les attitudes,

80. Antonine Maillet, Rabelais et les traditions populaires, op. cit., p. 58.

81. Ibid.

82. Jean-Claude Dupont, Contes et légendes, op. cit., p. 75.

83. William Lynwood Montell et Barbara Allen, From Memory to History: Using Oral Sources in Local Historical Research, Nashville, American Association for State and Local History, 1981, p. 23. Ancelet et Labelle sont aussi d'avis que, dans la communication des récits oraux, la vérité historique est soumise à la vérité psychologique : Barry-Jean Ancelet, «And This Is No Damn Lie: Oral History in Story Form », dans International Journal of History, vol. 4, $\mathrm{n}^{\circ} 2$, 1983, p. 99-111; Ronald Labelle, "L'histoire orale et l'identité culturelle chez les Acadiens de la Nouvelle-Écosse », 
les sentiments ou les croyances exprimés ${ }^{84}$. Le point de vue personnel que Maillet donne aux personnages et aux événements décrits est déterminant puisque c'est l'interprétation, plutôt que les faits, qui joue le plus grand rôle dans la création et la transmission de récits traditionnels, que ce soit à l'oral ou à l'écrit. Pour Montell, "what people believe to be important is often as important as what actually happened ${ }^{85}$. Traités comme textes culturels, les récits oraux deviennent des documents uniques qui mettent en lumière les conceptions et les valeurs, c'est-à-dire la vision du monde d'un groupe ${ }^{86}$. Ainsi, les œuvres de Maillet, s'inspirant de la tradition orale, transmettent aussi les sentiments du peuple acadien face à leurs expériences de vie.

dans Les Cahiers de la Société historique acadienne, vol. 15, n 4, p. 141-149.

84. Id., p. 89.

85. Id., p. 21.

86. Barry-Jean Ancelet, « Deep Meanings in Small Places: Social and Community Values in the Oral Traditions of French Louisiana ", article inédit, remis à The American Folklore Society en 2004, p. 1. 